\title{
Mobile Applications and Algorithms to Facilitate Electric Vehicle Deployment
}

\author{
Yuhuan Du and Gustavo de Veciana \\ Department of Electrical and Computer Engineering, The University of Texas at Austin \\ Email:dyhuan123@gmail.com,gustavo@ece.utexas.edu
}

\begin{abstract}
Although electric vehicles are attracting increasing interest from consumers and automakers, the disadvantages associated with limited range and the current scarcity of public recharge stations have played a key role in limiting their large scale adoption. In this paper, we explore how information technologies might be used to mitigate 'range anxiety' and further strengthen the potential of electric vehicle integration with the renewable energy generation and storage. We motivate several mobile applications/services which would improve the ownership experience of electric vehicles and flexibility for energy providers. Our work leverages a previously proposed sensor platform for collecting travel-time and energy-usage data for a road network by a community of electric car drivers. Traveltime and energy-usage on a given road segment may exhibit substantial variability due to environmental and temporal factors, e.g., congestion, road's grade, AC on/off, etc. Such variability in turn, makes it difficult to accurately predict travel-times as well as the feasible range of a car given its current energy reserves. However, by collecting statistical data using cars/mobiles as probes one can quantify such uncertainty and develop complementary algorithms to counter the anxiety and time waste associated with such uncertainty. This paper develops the necessary (routing) algorithms to support these new classes of applications/services for electric vehicles.
\end{abstract}

\section{INTRODUCTION}

In the last few decades, the environmental impact of the petroleum-based transportation has led to renewed interest in electric transportation infrastructure. Not only is the operating cost of electric vehicles much less than that of traditional vehicles, but the possibility of integrating an electric fleet with the smart grid, using demand side management, to make better use of volatile renewable generation, makes them an attractive component in building an efficient smart grid.

Various companies have introduced hybrid electric vehicles (HEV) and pure electric vehicles (EV) to enter this emerging market [1], [2]. Electric vehicles suffer from two basic problems. First, they have limited range, due to the low energy density of batteries as compared to fuel used in internal combustion engines. In fact, most pure electric vehicles currently have a manufacturer estimated range of 100 miles or so. The range however can vary substantially depending on driving style and the environment. For example, the range of the Nissan LEAF varies between 47 and 138 miles depending drastically on the EV's speed, traffic conditions, road type or even the operation of the air conditioner [3].

This work was supported by the National Science Foundation under Award CNS-0915928.
Second, the recharging process can be slow and inconvenient [4], [5]. Recharging takes different amounts of time depending on the voltage "level" of chargers. A $220 \mathrm{~V}$ home charging station may need more than 10 hours to fully charge a battery, while a higher voltage public charging station may need 10 to 30 minutes to charge a battery that is half depleted. To make things worse, there are only a few charging stations as compared to the traditional gas stations. The scarcity of public charging stations means the electric vehicle owners need to keep in mind the reachability of charging infrastructure. Although $75 \%$ of Americans commute 40 miles or less round trip, anxiety associated with the limited range of pure EVs has been a sore point for adopters. Indeed, this is why hybrid options have been developed and marketed in the last few years.

Meanwhile, there are billions of automobiles worldwide and new automobiles are equipped with hundreds of sensors. Recent work has shown the feasibility of collecting traffic information using such sensors, see e.g. [6], [7] or using smartphones e.g. [8]. The basic mechanism is simple: data is collected from sensors (accelerometers and location) in phones or on appropriately outfitted vehicles, and transmitted and aggregated at servers on Internet, see Fig.1. Such frameworks provide a means to collect a lot of detailed information on traffic and/or the road infrastructure.

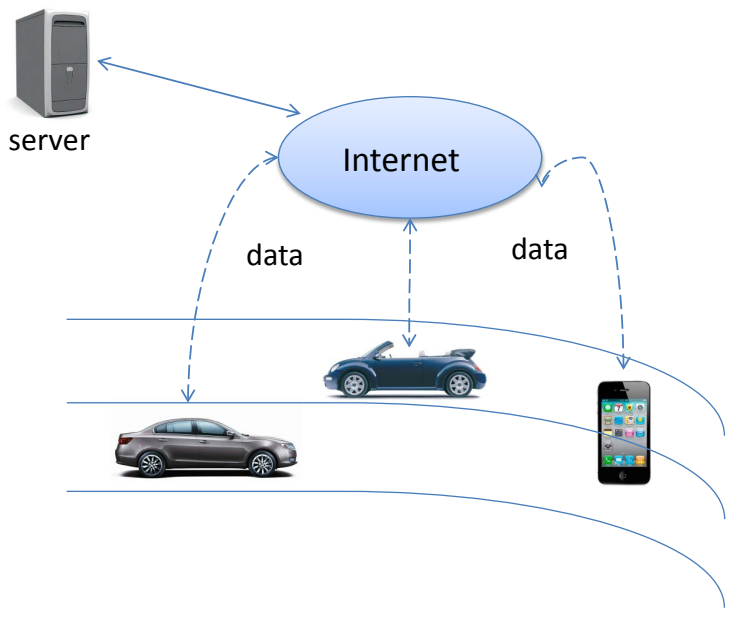

Fig. 1. Infrastructure to collect data

In this paper we focus on leveraging such sensor data to address various challenges associated with EVs, including 
mitigating the range anxiety by making more predictable estimates, better integrating the charging infrastructure, and other aspects associated with integrating EVs with the power grid. We will discuss the main applications in detail in Section II. Briefly speaking, these include: Charge Alert to alert drivers when they are running out of energy; Route Planner to find the best path considering time and energy requirements; Trip Planner and Charge Station Reservation System to help people plan a long distance EV trip; and Demand Scheduling and Energy Storage in Smart Grid to help integrate an electric fleet with the smart grid.

There are two basic challenges in making the above applications a reality: (1) collecting statistical data which reflects the mean and variability in drive-time and energy-usage on the roads to enable route computation and estimates that drivers can rely on; and (2) algorithmic challenges associated with computing such estimates and routes. Fortunately, as we discuss below in related work, there has already been substantial research and experience on building IT infrastructure to collect such data from a population of participant car drivers. There have also been efforts in analyzing and devising algorithms to address the computation of routes most likely to meet a driver's desired travel time related to a type of stochastic shortest path problems. This paper builds on both of these contributions to address new algorithmic challenges presented by the electric vehicles. We shall thus devote some time to related work.

\section{A. Related Work}

Data collection from a population of vehicles. The underlying data collection infrastructure for our work comes from [6]. This paper proposes CarTel, a mobile sensor computing system designed to collect, process and visualize data from sensors on vehicles. The collected data is transferred back to servers permitting the development of applications based on diverse types of queries. In a subsequent paper, [8] proposed a framework which uses sensors in smartphones to efficiently collect information from a community of users. The framework is fairly generic and intended to permit the collection of statistical information on the car drive-times on road segments as well as facilitate the identification of other characteristics of the road ways, e.g., presence of pot holes by recognizing the associated car drive patterns from inertial sensors. Clearly the same infrastructure can be used to collect the data needed for electric vehicles. For example cars equipped with energy sensors, could be used to collect statistical data associated with energy expenditures on road segments, implicitly capturing variability associated with fluctuations in traffic and/or timeof-day congestion, uphill/downhill characteristics, car/battery types, and/or driver style. In this paper we will assume that using the same type of infrastructure, as well of post processing of collected data, that distributions for the drive time and energy expenditures on segments of a road network are available, representing the typical scenario for drivers at a particular time of day.

Algorithm development: stochastic shortest path. With regard to algorithm development, a work closely related to this paper is [9]. The work is motivated by the following observation. Due to the uncertainty associated with drive times along a given route, 'anxious' drivers tend to budget much more time to make a trip than necessary, which leads to an overall substantial cost in wasted time eventually waiting at the destination etc. Thus, the critical problem in planning a route, is not to find the shortest path in terms of distance, but to find the path which is most likely to meet a target deadline, e.g., if one needs to make it to the airport by some time which is the best path? Doing so requires modeling the variability of the constituent road segments as well as determining an overall path whose aggregate distribution is the most favorable. In the above mentioned paper, travel time of every road segment is modeled by a random variable and paths are determined to maximize the probability that the total path travel time does not exceed a given target, and risks are explicitly assessed. This allows a driver/user of the application to define his level of tolerance to excess delay, and in turn the algorithm to take that into account in determining an appropriate path. In this paper we further consider the problem of 'range anxiety' associated with EV. This introduces problems which involve tradeoffs between uncertain drive-time and energy expenditures. We can express such tradeoffs in terms of additive functions of both time and energy and/or (probabilistic) constraints on some of the variables. Algorithmically in this paper we focus on two problems driven by energy-time tradeoffs: shortest path problem with constraints, and then to address uncertainty, extend this to stochastic shortest path with chance constraint problem. Researchers have already worked on shortest path problems with constraints in particular fields, e.g. [10] and stochastic shortest path problem, e.g. [9]. However, we will address more general problems which are related to electric vehicle-related services we envisage.

\section{B. Our Contributions}

In this paper we propose new types of services/applications for plugin electric vehicles leveraging information technology infrastructure. These services can make the ownership of electric vehicles a better experience and a competitive choice as compared to traditional petroleum-based transportation and less costly than hybrid EVs. The services can also serve as a basis for other innovative mechanisms for EV based energy demand scheduling and buffering in smart grid. Data collection to enable the design of such systems can easily be carried out via either vehicle or mobile-phone based infrastructures, examples of which were discussed above. Our focus is also on the algorithmic side of things in particular in looking to mitigate uncertainty in drive-time and energy expenditures, and thus the anxieties associated with these. We propose a new set of algorithms to realize tradeoffs, amongst drive-time, energy expenditures and risks of failing to meet users' needs.

We use a graph-based model in which the edges (corresponding to road segments) have multiple associated parameters which can either be constant values or random variables with known distribution. We start with a simple problem, 
where each edge has constant parameters associated with the expected travel time and energy consumption and a path cost function is a convex function of the overall path's travel time and energy consumption. We convert this problem to one of solving a traditional shortest path problem with linear path cost function. We then introduce constraints on this problem, which could, for example, correspond to requirements on the total travel time or energy expenditure. To determine shortest paths which also satisfy such constraints, we propose in iterative algorithm that at each step maintaining a onedimensional "path feasibility" vector and modify Dijkstra's algorithm accordingly.

Next we consider models which capture the role of uncertainty in such a network, by studying stochastic shortest path problems which, for example, account for variability in travel time and energy consumption. Our starting point is the stochastic shortest path (SSP) problem considered in [9] but we introduce chance constraints. In particular we model the travel time and energy expenditures associated with the graph's edges as random variables and set the goal of maximizing the probability of reaching the destination within a deadline while simultaneously meeting a chance constraint on the energy consumption on the selected path. In other words finding a path that meets a risk requirement on energy depletion, and at the same time maximizes the likelihood of arriving on time. We consider the case where random variables are normally distributed, which is likely a reasonable model in most cases of interest. In both cases, our algorithms have a worst case running time $O\left(n^{(\log n)}\right)$, where $n$ is the number of nodes in the road way graph model described below.

In summary the paper advances the state-of-the-art, by introducing a framework to enable the computation of reliable estimates for the distances EVs with limited battery resources can travel. This is achieved by collecting travel-time and energy expenditure statistics from a community/network of in-car or car bound mobile phone sensors and devising a new classes of algorithms to support path computation.

\section{Paper Organization}

This paper is organized as follows: Section II describes our proposed applications. Section III introduces our system model. Section IV develops our solution to the deterministic shortest path problem with constraints while Section V addresses the stochastic case. Section VI concludes the paper and points to some future work.

\section{AppliCATIONS}

This section introduces several mobile based applications for EVs and associated challenges.

Charge Alert. Because of the current scarcity of electric charging stations, it is possible for an EV to inadvertently run out of energy without access to a closeby public charging station. To avoid this a 'charge alert' application can be devised which leverages a priori knowledge of the available charging stations in a region and likely energy consumption on different road segments to alert the driver if there is trouble ahead. The key element in such a system would be the ability to accurately estimate the energy expenditures to reach the 'closest' charging station given the current location and/or desired destination of a vehicle. Given the uncertainty involved with driving relatively long distances (e.g., until the next charge point) and variability in congestion and possibly driver style, car energy expenditures, the driver should be alerted in a reliable manner as to the risk, without at the same time being too conservative and thus seriously limiting flexibility.

Route Planner. For electric vehicles the shortest path may not always be the most energy efficient. For example, driving on a highway involves cruising at higher speed but energy consumption increases dramatically with speed. If a car's battery is close to depleted, it may be preferable to take local roads where one can drive more slowly, and to avoid steep hills. Leveraging the above mentioned information one can design a route planner that suggests the best route based on different requirements. If the driver wants to go from $s$ to $d$, and has an energy budget $e_{0}$, the application can choose a path that achieves a desirable tradeoff between time and energy consumption. For example, a path with a high likelihood of reaching the destination within the time deadline, and negligible risk of energy depletion. This involves solving a shortest path problem with constraints.

Trip Planner and Charge Station Reservation System. Although most electric vehicles are used in and around town, EV owners may occasionally wish to travel long distances. In this case recharging along the way will be unavoidable and thus should play a key role in planning the overall trip, with charging stops along the way. Further since recharging an electric vehicle can take a long time [4] and the facilities are limited, particularly in rural areas, a good trip plan might include making a reservation to charge the car ahead of time at a likely arrival time, so that upon arrival a charging spot is available. This could help the customers avoid long waits in accessing limited resources and help the charging station providers increase profits.

Demand Scheduling and Energy Storage in Smart Grid. An electric grid shared by a large population of electric vehicles presents new opportunities for integrating renewable generation. Indeed renewable generation, e.g., wind and solar, may be highly variable, but when a large population of EVs are plugged in (e.g., at work/home/public parking with chargers), one can schedule their aggregate load on the network to match the generation. Such scheduling demand could in principle take into account constraints placed by drivers on the timing, and next destination to ensure drivers do not subsequently suffer by not being able to reach their desired destination. Additionally, more aggressive concepts involve possibly allowing the grid to subsequently take energy from the cars. Although possibly not efficient, this effectively allows storage of energy. Again discharging of a car's battery, should be subject to the drivers near term goals, e.g., will need to go home, so reasonable energy budget should be left to allow for it. Once again the essence here is to ensure energy reserves 
are present to reliably guarantee the vehicle will be able to reach the desired destination, e.g., home or work.

Next we will describe the system model and algorithms to implement such applications.

\section{SySTEM MODEL}

\section{A. Model Abstraction}

We model the map of a regions a graph. Intersections are nodes and road segments are modeled as edges. Each edge is associated with several non-negative parameters which are either constants or random variables with known distribution. In this paper the parameters correspond to travel time and energy consumption. The constants will represent the expected values while the random variables represent the travel time or energy consumption when different vehicles pass the same road segment at a given time of day. The problems of interest are expressed in terms of this graph. More explicitly, consider a graph $G=(V, E)$, with $|V|=n$ nodes and $|E|=m$ edges. We have a source $s$ and a destination $d, s, d \in V$. Formally speaking, a path $p$ in graph $G$ is a sequence of nodes such that from each of its nodes there is an edge to the next node in the sequence. $P_{s d}$ represents the set of all the paths from $s$ to $d$. Each edge $i$ has two non-negative parameters: denoted $e_{i}$ and $t_{i}$ if constants or $E_{i}$ and $T_{i}$ if random variables, where ' $e_{i}$ ', ' $E_{i}$ ' are energy related parameters and ' $t_{i}$ ', ' $T_{i}$ ' are associated with travel times. For random variables, we assume $E_{i} \sim N\left(\mu_{e}(i), \sigma_{e}^{2}(i)\right), T_{i} \sim N\left(\mu_{t}(i), \sigma_{t}^{2}(i)\right)$. The problem of interest will be either to find the shortest path subject to a constraint on the path or to find stochastic shortest path subject to a chance constraint.

\section{B. Previous Results}

We shall introduce some definitions and results from [9], [11] which serve as a starting point for our work.

Definition 1: A set $S$ is said to be convex if for all $x, y \in S$ and $\alpha \in[0,1], \alpha x+(1-\alpha) y$ is in $S$.

Definition 2: The convex hull for a set of points $X$ is the smallest convex set containing $X$.

Definition 3: Let $C$ be a convex set. A function $f: C \rightarrow \mathbb{R}$ is quasi-convex if all its lower level sets, i.e. $L_{\gamma}=\{x \mid x \in$ $C, f(x) \leq \gamma\}$ for $\gamma \in \mathbb{R}$, are convex.

Definition 4: A point $x$ is an extreme point of a convex set $C$ if it cannot be represented as a convex combination of two other points in $C$, i.e., $x$ is an extreme point if when

$x=\alpha y+(1-\alpha) z$ for $y, z \in C, \alpha \in(0,1)$ then $y=z=x$

Definition 5: The dominant of a set $S$ where $S \subset \mathbb{R}^{m}$ is the set of all points that are greater than a point in $S$,

$$
\left\{x \in R^{m} \mid x \geq y \text { for some } y \in S\right\},
$$

$S$ need not be convex.

Theorem 1: Let $C \subset \mathbb{R}^{2}$ be a convex set, and $f: \mathbb{R}^{2} \rightarrow$ $\mathbb{R}$ be a quasi-convex function which is monotone increasing (decreasing) in both parameters, then $f$ attains the minimum (maximum) over $C$ at some extreme point of the dominant of C.
Let us consider our graph-based model where we are given a source $s$ and destination $d$ in the graph, and each edge $i$ has two non-negative parameters $e_{i}, t_{i}$, associated with it.

Definition 6: Suppose each edge $i$ is associated with a parameter dependent weight $\lambda e_{i}+t_{i}$ where $\lambda \in \mathbb{R}^{+}$. Clearly the graph has a parameter $\lambda$ dependent shortest path from $s$ to $d$. We define breakpoints as the values of $\lambda$ at which the shortest path changes. The parametric shortest path problem is to determine all such breakpoints.

Theorem 2: (Carstensen [11]) There are at most $n^{O(\log n)}$ breakpoints for the parametric shortest path problem with parameter lengths $\lambda e_{i}+t_{i}$, where $i \in E$ and $\lambda \in \mathbb{R}^{+}$.

Definition 7: The projection of a path $p$ onto the plane spanned by $\sum_{i \in p} e_{i}$ and $\sum_{i \in p} t_{i}$ (or projection of path $p$ for short) is the point with coordinate $\left(\sum_{i \in p} e_{i}, \sum_{i \in p} t_{i}\right)$. The projection of all the paths is the point set which contains the projection of every path from $s$ to $d$.

Theorem 3: Let $X$ denote the projection of all the paths (or all the feasible paths) from $s$ to $d$. As shown in Fig. 2, for each $\lambda$ we can find a shortest path and the projection of this path is an extreme point of the dominant of the convex hull of $X$. Further, [9] shows that there is a one-to-one correspondence between the extreme points of the dominant of the convex hull of $X$ and the breakpoints of the parametric shortest path problem with parameter length $\lambda e_{i}+t_{i}$ and the time complexity to find all extreme points is $O\left(n^{(\log n)}\right)$.

\section{Shortest Path With Constraints}

This section introduces the main idea to implement Route Planner application. We begin by introducing a class of problems and associated algorithms that address multiparamter shortest path problems on graphs, where edge parameters do not exhibit uncertainty or are based on means.

\section{A. Multi-Parameter Shortest Path Problem without Con- straints}

Let us start by considering a shortest path problem without constraints, but with multiple edge weights on which the cost function depends.

We are motivated by scenarios where different people may wish to achieve different tradeoffs between energy and travel time. Some want to minimize travel time, some want to minimize energy consumption and others may want to minimize the sum of travel time and square root of energy consumption. The cost functions of interest here are monotone increasing in total energy and travel time. Further, we assume the cost function is quasi-convex, which is reasonable in most cases. This problem is also the basis for more complicated problems considered in the sequel.

Consider a graph $G=(V, E)$, with $|V|=n$ nodes and $|E|=m$ edges. We have a source $s$ and a destination $d$. Each edge $i$ has two weights $e_{i}$ and $t_{i}$. In this subsection, we consider the following problem:

Multi-parameter Shortest Path (MSP) 


$$
\underset{p}{\arg \min }\left\{f\left(\sum_{i \in p} e_{i}, \sum_{i \in p} t_{i}\right) \mid p \in P_{s d}\right\}
$$

where $P_{s d}$ denotes the set of paths between $s$ and $d$ and $f$ : $\mathbb{R}^{2} \rightarrow \mathbb{R}$ is a quasi-convex and monotone increasing function.

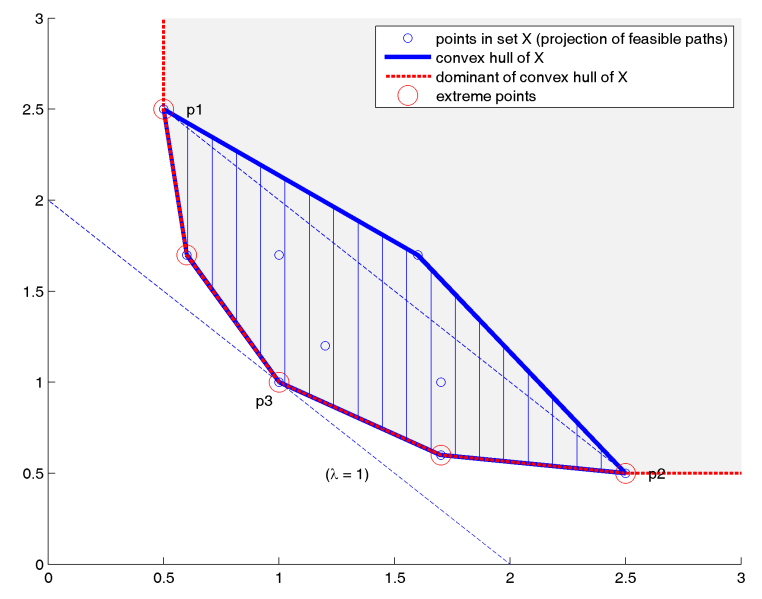

Fig. 2. The extreme points of the dominant of the convex hull of the projection of all the feasible paths from $s$ to $d$ and the process to get $p_{3}$ after obtaining $p_{1}$ and $p_{2}$.

If $f$ is not linear, this problem can not be directly solved via Dijkstra's algorithm. However, by Theorem 1 in Section III.B, the solution must be associated with an extreme point of the dominant of the convex hull of the projection of the feasible paths $P_{s d}$. In turn, each extreme point is the projection of the solution to a shortest path problem

$$
\underset{p}{\arg \min }\left\{\sum_{i \in p}\left(\lambda e_{i}+t_{i}\right) \mid p \in P_{s d}\right\}
$$

for some $\lambda \in \mathbb{R}$. Such problems are easily solved via Dijkstra's algorithm.

By letting $\lambda$ vary from 0 to $+\infty$, one can determine all the extreme points. As shown in Fig. 2, we start from two endpoints: the leftmost point $p_{1}$, which corresponds to the solution of $\min _{p}\left\{\sum_{i \in p} e_{i} \mid p \in P_{s d}\right\}$ (or $\lambda_{1} \rightarrow \infty$ ) and the rightmost point $p_{2}$, which corresponds to the solution of $\min _{p}\left\{\sum_{i \in p} t_{i} \mid p \in P_{s d}\right\}$ (or $\lambda_{2}=0$ ). Suppose $p_{1}=\left(p_{1 e}, p_{1 t}\right)$ and $p_{2}=\left(p_{2 e}, p_{2 t}\right)$. Let $\lambda_{3}=-\frac{p_{1 t}-p_{2 t}}{p_{1 e}-p_{2 e}}$, which represents the inverse of the slope of the line passing $p_{1}$ and $p_{2}$, we get an extreme point $p_{3}$. If $p_{3}$ is the same as $p_{1}$, this means all the $\lambda$ between $\lambda_{1}$ and $\lambda_{3}$ will obtain the same extreme point. If $p_{3}$ is different from $p_{1}$, we can repeat this to find a new extreme point between $p_{1}$ and $p_{3}$. By repeating this process one can find all the extreme points.

By Theorem 3 the total time complexity is $O\left(n^{(\log n)}\right)$.

After obtaining all the extreme points, one need only evaluate $f(\cdot, \cdot)$ at these points to find the solution to the problem.

\section{B. Shortest Path Problem with Constraints}

Next we introduce the problem with constraints. In our context, for example, the constraint may be not running out of energy for the selected path or the total travel time not exceeding a deadline. By solving this we can implement a basic version of Route Planner service.

Consider the system model described above with constants as edge weights. In this case we consider the Multi-parameter Shortest Path with Constraints (MSPC) problem:

$$
\underset{p}{\arg \min }\left\{f\left(\sum_{i \in p} e_{i}, \sum_{i \in p} t_{i}\right) \mid p \in P_{s d}, \quad \sum_{i \in p} g\left(e_{i}, t_{i}\right)<\delta\right\},
$$

where $f: \mathbb{R}^{2} \rightarrow \mathbb{R}$ is quasi-convex and monotone increasing in both parameters and $g: \mathbb{R}^{2} \rightarrow \mathbb{R}$ can be any function.

As in the previous section, the solution to MSPC is also associated with an extreme point. The problem can be solved by first solving

$$
\underset{p}{\arg \min }\left\{\sum_{i \in p}\left(\lambda e_{i}+t_{i}\right) \mid p \in P_{s d}, \quad \sum_{i \in p} g\left(e_{i}, t_{i}\right)<\delta\right\} .
$$

If we can solve this, we can follow the same approach as in the previous subsection. Specifically, we can first vary the value of $\lambda$ to find all the "feasible" extreme points and then substitute them in $f$ to find the best solution.

However, the parametric shortest path problem with constraints is different from the problem considered previously, which could be solved using Dijkstra's algorithm. The shortest path found by Dijkstra's algorithm may not be "feasible". Here we propose a simple modification of Dijkstra's algorithm to solve the shortest path problem with constraints.

To describe our approach, we use $M F P_{s d}$ to represent the feasible path from $s$ to $d$ with minimal cost function. In Dijkstra's algorithm, we actually explore the shortest paths from $s$ to all other nodes until we find the shortest path from $s$ to $d$. However in our algorithm, we explore the shortest "possibly feasible" shortest paths to all other nodes until we find $M F P_{s d}$.

Note that a path $p_{s t}$ from $s$ to $t$ is possibly part of $M F P_{s d}$, i.e. possibly feasible, if and only if the summation of cost on $p_{s t}$ with regard to $g$ and minimal cost with regard to $g$ needed to travel from $t$ to $d$ is less than $\delta$. At the same time, since we want to minimize the cost with regard to $\lambda e_{i}+t_{i}$, we should implement Dijkstra's algorithm by considering the cost as $\lambda e_{i}+t_{i}$ but check the feasibility at each step according to the above observation.

The algorithm details are described in Algorithm 1.

We note that the one-dimensional matrix LengthToDestFrom[] is the key to dealing with the problem with constraints, where LengthToDestFrom $[j]$ records the shortest length from $j$ to $d$ by considering the length of edge $i$ as $g\left(e_{i}, t_{i}\right)$. As long as we can build LengthToDestFrom[] in the initialization step of the algorithm, the problem with constraints can be solved. If there were more than one constraint, we need only keep track of different LengthToDestFrom[] associated with different constraints. 


\section{Stochastic Shortest Path With "Chance" CONSTRAINTS}

Algorithm 1 Shortest Path with Constraints

1: Initialization: the shortest path tree $S P T$ is null. Use Dijkstra's algorithm to build the one-dimension matrix LengthToDestFrom[], where LengthToDestFrom $[j]$ records the shortest length from node $j$ to $d$ by considering the length of edge $i$ as $g\left(e_{i}, t_{i}\right)$.

2: $\quad$ If LengthToDestFrom $[s] \geq \delta$, there will be no solution for this problem. Go to the step 10.

- Otherwise, continue to step 3.

3: For each edge $i$, compute $f_{i}=\left(\lambda e_{i}+t_{i}\right), g_{i}=g\left(e_{i}, t_{i}\right)$.

4: Assign to every node a fLength value and a gLength value: set them to zero for $s$ and to infinity for all other nodes.

5: Mark all nodes unvisited. Set $s$ as current. Create a set of the unvisited nodes called the unvisited set consisting of all the nodes.

6: For the current node $a$, consider all of its unvisited neighbors one by one in the following way:

For a neighbor $i$, first compute its $g$ Length value tempi, i.e. the $g$ Length value of node $a$ plus $g_{k}$ for the edge $k$ from $a$ to $i$.

- If tempi + LengthToDestFrom $[i]<\delta$, compute its $f$ Length value and update fLength and gLength if we get a smaller $f$ Length. For example, if the current node $a$ has $f$ Length $=6$, and the edge $k$ connecting it with neighbor $i$ has $f_{k}=2$, then $f$ Length value of $i$ (through $a$ ) will be $6+2=8$. If this value is less than the previously recorded fLength value, then overwrite fLength and gLength (with tempi) for node $i$. After these, go to the next neighbor of $a$. If this is the last neighbor, go to step 7.

- Otherwise, directly go to the next neighbor of $a$. If this is the last neighbor, go to step 7 .

Note, even though a neighbor has been examined, it is not marked as visited at this time, and it remains in the unvisited set.

7: When we are done considering all of the neighbors of the current node, mark the current node as visited, remove it from the unvisited set and add it to the $S P T$.

8: $\quad$ - If all the unvisited nodes have infinity f Length value, there is no solution for this problem. Go to step 10 .

- Otherwise, set the unvisited node marked with the smallest $f$ Length value as the next "current node"

- If the current node is $d$, add it to the $S P T$, then the problem is solved. Go to step 9 .

- If the current node is not $d$, go back to step 6 .

9: The problem is solved. Refer to the $S P T$ and output the shortest path with constraints from $s$ to $d$.

10: There is no solution for this problem.
We now will consider the stochastic shortest path problem. Since factors such as travel speed, congestion and road grade may influence the travel time and energy consumption of electric vehicles traversing a given road segment, it is meaningful to model both the travel time and energy consumption associated with edges as random variables.

The stochastic shortest path (SSP) problem without constraints was considered in [9]. Here we consider a version with "chance" constraints associated with energy or travel time requirements.

Such a problem arises naturally in practice when a user wants to maximize the probability of arriving on time while ensuring the probability of expending too much energy remains low, i.e. on budget.

Consider the system model described above with random variables as edge parameters. The random variables associated with different edges are assumed to be independent of each other, i.e., $E_{1}$ and $E_{2}$ are independent, but $E_{1}$ and $T_{1}$ need not be independent. Suppose we have a time deadline $\alpha$, i.e. we want to find a path maximizing the probability that we reach the destination within $\alpha$ minutes. Further, there is a "chance" constraint $\delta \in(0,1)$ that we consume no more than $\beta$ units of energy along the path. Formally, the problem of Stochastic Shortest Path with Constraints (SSPC) is to solve

$$
\underset{p}{\arg \max }\left\{P\left(\sum_{i \in p} T_{i} \leq \alpha\right) \mid p \in P_{s d}, P\left(\sum_{i \in p} E_{i} \leq \beta\right)>\delta\right\},
$$

where $\alpha$ represents a time deadline and $\beta$ represents an energy budget for the trip that should not be exceeded, such as the initial battery level.

In Section III, we assumed that for each edge $i, E_{i} \sim$ $N\left(\mu_{e}(i), \sigma_{e}^{2}(i)\right), T_{i} \sim N\left(\mu_{t}(i), \sigma_{t}^{2}(i)\right)$, and for any path $p$, let $m_{t}(p)=\sum_{i \in p} \mu_{t}(i)$ and $v_{t}(p)=\sum_{i \in p} \sigma_{t}^{2}(i)$, similarly we define $m_{e}(p)$ and $v_{e}(p)$. Now

$$
\begin{aligned}
P\left(\sum_{i \in p} T_{i} \leq \alpha\right) & =P\left(\frac{\sum_{i \in p} T_{i}-m_{t}(p)}{\sqrt{v_{t}(p)}} \leq \frac{\alpha-m_{t}(p)}{\sqrt{v_{t}(p)}}\right) \\
& =\Phi\left(\frac{\alpha-m_{t}(p)}{\sqrt{v_{t}(p)}}\right),
\end{aligned}
$$

where $\Phi$ denotes the cumulative distribution function (CDF) of the standard normal distribution and

$$
\begin{aligned}
P\left(\sum_{i \in p} E_{i} \leq \beta\right) & =P\left(\frac{\sum_{i \in p} E_{i}-m_{e}(p)}{\sqrt{v_{e}(p)}} \leq \frac{\beta-m_{e}(p)}{\sqrt{v_{e}(p)}}\right) \\
& =\Phi\left(\frac{\beta-m_{e}(p)}{\sqrt{v_{e}(p)}}\right) .
\end{aligned}
$$

Since $\Phi$ is monotone increasing, SSPC is equivalent to

$$
\underset{p}{\arg \max }\left\{\frac{\alpha-m_{t}(p)}{\sqrt{v_{t}(p)}} \mid p \in P_{s d}, \Phi\left(\frac{\beta-m_{e}(p)}{\sqrt{v_{e}(p)}}\right)>\delta\right\},
$$


which in turn is equivalent to

$$
\underset{p}{\arg \max }\left\{\frac{\alpha-m_{t}(p)}{\sqrt{v_{t}(p)}} \mid p \in P_{s d}, \frac{\beta-m_{e}(p)}{\sqrt{v_{e}(p)}}>\phi_{\delta}\right\},
$$

where $\phi_{\delta}$ is a constant such that $\Phi\left(\phi_{\delta}\right)=\delta$.

If $\alpha<\min _{p}\left\{m_{t}(p) \mid p \in P_{s d}\right\}$, it means the time deadline is even smaller than the mean time needed to get to the destination $d$. This is not the case of interest here, we will assume $\alpha \geq \min _{p}\left\{m_{t}(p) \mid p \in P_{s d}\right\}$.

Let $f(m, v)=\frac{\alpha-m}{\sqrt{v}}$ and note that this is a quasi-convex, monotone decreasing function, so by Theorem 1 , this problem can once again be solved by finding the "feasible" extreme points, i.e., solving

$$
\underset{p}{\arg \min }\left\{\lambda m_{t}(p)+v_{t}(p) \mid p \in P_{s d}, \frac{\beta-m_{e}(p)}{\sqrt{v_{e}(p)}}>\phi_{\delta}\right\},
$$

for $\lambda \in \mathbb{R}^{+}$.

This is equivalent to

$$
\begin{gathered}
\underset{p \in P_{s d}}{\arg \min }\left\{\lambda m_{t}(p)+v_{t}(p)\right\} \\
\text { s. t. } m_{e}(p)+\phi_{\delta} \times \sqrt{v_{e}(p)}<\beta
\end{gathered}
$$

Using the algorithm we proposed to solve the MSPC, we can build the matrix LengthToDestFrom[] associated with the constraint to tackle the problem, i.e. to check the feasibility at each step. Building the matrix LengthToDestFrom[] is equivalent to solving

$$
\min _{p}\left\{m_{e}(p)+\phi_{\delta} \times \sqrt{v_{e}(p)} \mid p \in P_{j d}\right\},
$$

for each node $j$.

Let $h(m, v)=m+\phi_{\delta} \times \sqrt{v}$ and note that $h$ is a quasi convex and monotone increasing function, thus we recognize that this problem is similar to MSP that was tackled in Section IV.A. Using the solutions to MSP, we need to solve

$$
\underset{p}{\arg \min }\left\{\lambda m_{e}(p)+v_{e}(p) \mid p \in P_{j d}\right\},
$$

for each node $j$ and for "all" $\lambda$ from 0 to $+\infty$.

Next we analyze the complexity of the solutions. Since there are $n$ nodes in the graph and for each node $j$ the time complexity is $O\left(n^{(\log n)}\right)$, the total time complexity is $O\left(n \times n^{(\log n)}\right)=O\left(n^{1+\log n}\right)=O\left(n^{(\log n)}\right)$.

In practice, we find that the actual computation is much less than $n$ times that for a single node. Since the graph is undirected, the problem is equivalent to considering $d$ as the source and node $j$ varying across all possible destinations. When we solve the problem for a fixed node $j$ which is far from $d$ and a fixed $\lambda$, we have also solved it for many other nodes and the same $\lambda$ because we have explored many other nodes as we find the shortest path from $d$ to $j$.

\section{CONCLUSION AND Future WORK}

In this paper we showed how we could leverage sensor data regarding variable vehicles drive times and energy expenditures on a road network to address some of the current challenges associated with EVs: range anxiety and scarcity of public charging stations. By considering energy constraints in the problem setup, our algorithms leverage measurements to provide a basis for reliable range-energy estimates and planning for energy-feasible routes, which would in part mitigate range anxiety for EV owners and also enable integrating EVs with a smart grid. Systems and applications built on these would judiciously manage energy needs and availability, while meeting drivers and consumers goals. There are several interesting topics for future work. Trip planning over longer ranges requires determining when and where to stop for $\mathrm{EV}$ recharge. It would be nice to integrate this problem with our existing algorithms. Our premise is that an electric vehicle fleet can benefit significantly from information technologies enabling their deployment, yet this is still a relatively unexplored area. As such we have access to only limited data. Obtaining data to conduct an experimental evaluation of the proposed ideas on a mid-size EV fleet is our goal for future work.

\section{ACKNOWLEDGMENT}

We thank Yang Guo and Zheng Lu for their help with this paper.

\section{REFERENCES}

[1] "Electric vehicle in wikipedia." [Online]. Available: http://en.wikipedia.org/wiki/Electric_vehicle

[2] C. Chan, "The state of the art of electric and hybrid vehicles," in Proceedings of the IEEE, vol. 90, August 2002, pp. 247-275.

[3] "Extending range of electric vehicles by 10 percent with gpslike device." [Online]. Available: http://phys.org/news/2012-07-rangeelectric-vehicles-percent-gps-like.html

[4] K. Kowalenko, "Going electric: Things to consider," The Institute, IEEE, September 2011.

[5] C. Botsford and A. Szczepanek, "Fast charging vs. slow charging: Pros and cons for the new age of electric vehicles," EVS24 Stavanger, Norway, May 13-16 2009

[6] B. Hull, V. Bychkovsky, Y. Zhang, K. Chen, M. Goraczko, A. Miu, E. Shih, H. Balakrishnan, and S. Madden, "Cartel: a distributed mobile sensor computing system," in Proceedings of the 4th international conference on Embedded networked sensor systems, November 1-3 2006.

[7] J. Eriksson, L. Girod, B. Hull, R. Newton, S. Madden, and H. Balakrishnan, "The pothole patrol: Using a mobile sensor network for road surface monitoring," ACM MobiSys, June 17-20 2008.

[8] A. Thiagarajan, L. Ravindranath, K. LaCurts, S. Madden, H. Balakrishnan, S. Toledo, and J. Eriksson, "Vtrack: accurate, energy-aware road traffic delay estimation using mobile phones," in Proceedings of the 7th ACM Conference on Embedded Networked Sensor Systems, November 4-6 2009.

[9] E. Nikolova, J. A. Kelner, M. Brand, and M. Mitzenmacher, "Stochastic shortest paths via quasi-convex maximization," in Algorithms ESA 2006 , ser. Lecture Notes in Computer Science. Springer Berlin / Heidelberg, 2006, vol. 4168, pp. 552-563.

[10] R. J. Szczerba, P. Galkowski, I. S. Glickstein, and N. Ternullo, "Robust algorithm for real-time route planning," IEEE Transactions on Aerospace and Electronic Systems, vol. 36, no. 3, pp. 869-878, July 2000.

[11] P. Carstensen, "The complexity of some problems in parametric linear and combinatorial programming," Ph.D. Thesis, Mathematics Dept., U. of Michigan, Ann Arbor, Mich., 1983. 